\title{
Long noncoding RNA MYLK-AS1 promotes growth and invasion of hepatocellular carcinoma through the EGFR/HER2-ERK1/2 signaling pathway
}

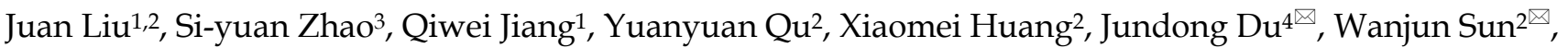 \\ and Qinong $\mathrm{Ye}^{1 凶}$ \\ 1. Department of Medical Molecular Biology, Beijing Institute of Biotechnology, Beijing 100850, China \\ 2. Department of Hematology, PLA Rocket Force Characteristic Medical Center, Beijing 100088, China. \\ 3. Medical unit, 91638 Troops, PLA, Beijing 102202, China. \\ 4. Department of Surgery, Hebei Yanda Hospital, Hebei 065201, China. \\ $\triangle$ Corresponding authors: Qinong Ye, Department of Medical Molecular Biology, Beijing Institute of Biotechnology, Beijing 100850, China. Phone: \\ 8610-66931830; Fax: 8610-88271216; E-mail: yeqn66@yahoo.com; Wanjun Sun, Department of Hematology, PLA Rocket Force Characteristic Medical Center, \\ Beijing 100088, China. Phone: 8610-66343351; Fax: 8610-66343351; E-mail: sunwanjun7471@126.com. Jundong Du, Department of Surgery, Hebei Yanda Hospital, \\ Hebei 065201, China. Phone: 86316-3306242; Fax: 86316-3306242; E-mail: Frank304301@163.com.
}

(c) The author(s). This is an open access article distributed under the terms of the Creative Commons Attribution License (https://creativecommons.org/licenses/by/4.0/). See http:/ /ivyspring.com/terms for full terms and conditions.

Received: 2019.12.15; Accepted: 2020.03.24; Published: 2020.04.27

\begin{abstract}
The epidermal growth factor receptor (EGFR) family members EGFR and HER2 play pivotal roles in oncogenesis and tumor progression. Anticancer drugs targeting EGFR and HER2 have been developed. Long noncoding RNAs (IncRNAs) have been reported to regulate cancer development and progression through signaling pathways. However, IncRNAs that regulate EGFR and HER2 expression remain unknown. Here, we show that IncRNA myosin light chain kinase-antisense RNA 1 (MYLK-AS1) promotes EGFR and HER2 expression and activates their downstream signaling pathway. MYLK-ASI increases hepatocellular carcinoma (HCC) cell proliferation, migration, and invasion in vitro. Consistently, MYLK-ASI knockdown hinders tumor growth in vivo. Mechanistically, MYLK-ASI enhances HCC cell proliferation, migration, and invasion through stimulating the EGFR/HER2-extracellular signal-regulated kinase $1 / 2$ (ERK1/2) signaling pathway. In addition, MYLK-AS1 is overexpressed in HCC patients and negatively correlated with HCC prognosis. Thus, MYLK-AS1 is an upstream regulator of EGFR/HER2, and acts as an oncogene, suggesting an additional target for cancer therapeutics.
\end{abstract}

Key words: LncRNA, MYLK-AS1, hepatocellular carcinoma, EGFR, HER2

\section{Introduction}

The erythroblastic leukemia viral oncogene homolog (ErbB) family consists of the epidermal growth factor receptor (EGFR, also known as ErbB1), ErbB2 (also known as HER2), ErbB3 (HER3) and ErbB4 (HER4), all of which are structurally related receptor tyrosine kinases (RTKs) [1]. These proteins, especially EGFR/HER2, are usually activated in many malignant tumors, including hepatocellular carcinoma (HCC) [2,3]. Overexpression or activation of EGFR/HER2 or their downstream signaling pathways is closely related to drug resistance, which finally leads to poor outcomes of patients [4]. The main downstream signaling pathway of EGFR/HER2 includes the rat sarcoma (RAS)-RAF proto-oncogene serine/threonine protein kinase-mitogen-activated protein kinase kinase 1/2 (MEK1/2)-extracellular signal regulated kinase $1 / 2$ (ERK1/2) pathway controlling cancer development and progression [5-7]. Several approaches, including the development of antibodies and small molecule inhibitors, have been employed to target EGFR and HER2 receptors or EGFR/HER2-modulated effects for cancer therapy [8-11]. Although clinical studies reveal promising results when using antibodies or inhibiters of 
EGFR/HER2 or both approaches, many patients are resistant to these treatments by activating or overexpressing alternative pathways and present a more aggressive phenotype, with more migratory and invasive cells compared to the non-resistant tumors. Therefore, elucidating molecular mechanisms of regulating EGFR/HER2 and their downstream signaling pathways are urgently needed.

Long non-coding RNAs (LncRNAs) with a length longer than 200 nucleotides are a class of non-protein coding transcripts [12]. More and more studies indicate that lncRNAs play essential roles in oncogenesis and progression of HCC by acting on proteins, stabilizing mRNA, regulating miRNA, or signaling pathways [13-15]. HCC is a large proportion of tumors worldwide and China alone accounts for about $50 \%$ of the total liver cancer rates [16]. Despite great improvements in exploring molecular mechanisms of HCC progression and therapeutic treatments, the overall survival (OS) and recurrence-free survival (RFS) rates, and the 5-year survival rates are still comparatively low, which means that the underlying molecular mechanisms need to be deeply and comprehensively studied $[17,18]$. MYLK-AS1, locating at chromosome 3, is a kind of long non coding RNA with a length of $814 \mathrm{bp}$. MYLK-AS1 was originally reported in the National Institutes of Health Mammalian Gene Collection (MGC) Program to identify putative alternative promoters of human genes but with no detailed studies $[19,20]$. Very recently, the lncRNA expression-based risk score system for OS of HCC patients has been constructed based on six lncRNAs including MYLK-AS1 [21]. MYLK-AS1 was reported to be downregulated in 9 colon adenocarcinoma samples compared to paired adjacent normal tissues [22]. However, the biological function of MYLK-AS1 is unknown, and the clinical significance of MYLK-AS1 still requires to be further determined.

In the present study, we used gene set enrichment analysis (GSEA) to investigate lncRNAs involved in the EGFR/HER2-ERK1/2 signaling pathway, and identified MYLK-AS1 as an upstream regulatory factor of EGFR/HER2. In HCC patients, MYLK-AS1 is up-regulated, and negatively correlated with prognosis. MYLK-AS1 promotes growth and invasion of HCC through the EGFR/HER2-RASRAF-MEK1/2-ERK1/2 signaling pathway.

\section{Materials and methods}

\section{Data source}

The RNA-seq data and clinical information of HCC were downloaded from the TCGA data portal (https://tcga-data.nci.nih.gov/) with a total of 377 samples (377 tumor samples and 41 normal tissue samples adjacent to tumors). Of these, 369 patients had complete prognostic information. Sequence data were generated using the Illumina HiSeq 2000 RNA Sequencing platform. The data were downloaded in September, 2019.

\section{Functional enrichment analysis}

Based on lncRNAs and gene relationships calculated by the Pearson correlation coefficients, functional enrichment analysis for lncRNAs in the lncRNA signature set was performed using DAVID. Gene set enrichment analysis (GSEA) was conducted using GSEA 4.0.1 version. GSEA 4.0.1 was performed by the JAVA program (http://software. broadinstitute.org/gsea/downloads.jsp) using the MSigDB C2 canonical pathways gene set collection, which contains 1320 gene sets. Gene sets with a false discovery rate (FDR) value less than 0.05 after performing 1000 permutations were considered to be significantly enriched [23]. If most sequenced genes correlated with higher expressions of lncRNAs are enriched at the top of K-RAS signaling up gene set, we speculate that these lncRNAs are positively correlated with K-RAS signaling up.

\section{Plasmids, siRNA, shRNA, and lentiviruses}

The eukaryotic expression vectors with full length MYLK-AS1 were generated by inserting PCR-amplified fragments into pcDNA3.0 (Invitrogen) to overexpress MYLK-AS1. To knock down the expression of MYLK-AS1, ERK and EGFR, small interfering RNAs (siRNAs) were designed and synthesized by RiboBio. The sequences of siRNAs for MYLK-AS1 are 5'-CCTCTTAAATGCTATACTA-3', 5'-GTGCGGGATTGCTGATGAA-3', 5'-ACATTTCTG TAAAGATGAA-3', 5'-GTTCAAGTGCATCTTCCAG C-3', 5'-GAGAGACAAGAGCAGGACAG and CAGCCAGTGAGATTGGAAGC-3'. The sequences of siRNAs for ERK1/2 are 5'-GACCGGAUGUUAACCU UUA-3' (ERK1); and 5'-CCAGGAUACAGAUCUU AAA-3' (ERK2) [24]. The sequence of siRNA for EGFR is 5'-GAGGAAAUAUGUACUACGAdTdT-3' [25]. According to the MYLK-AS1 siRNAs, a short hairpin RNA (shRNA) sequence aiming for MYLK-AS1 was subcloned into pSIH-H1-Puro (System Biosciences). Lentiviruses were produced by co-transfection of HEK293T cells with recombinant lentivirus vectors and pPACK Packaging Plasmid Mix (System Biosciences) using Megatran reagent (Origene), and were used to infect target cells according to the manufacturers' instructions.

\section{Cell lines and drug treatments}

The human hepatoma cell lines, $\mathrm{HepG}_{2}$, BEL-7402, SMMC-7721, MHCC97-H, the human normal cell lines LO2 and HEK293T were purchased 
from American Type Culture Collection (ATCC), and their mycoplasma contaminations have previously been tested. Cells were usually cultured in DMEM containing $25 \mathrm{mM}$ glucose (Invitrogen) and 10\% fetal bovine serum (FBS) (Hyclone) in an atmosphere of $5 \%$ $\mathrm{CO}_{2}$ at $37^{\circ} \mathrm{C}$. Five micrograms of plasmids and $50-200$ $\mathrm{nM}$ siRNAs as indicated were transfected into cells using Lipofectamine 2000 reagent and Lipofectamine RNAiMAX, respectively, according to the manufacturers' instructions (Invitrogen and RiboBio). Cells with $70-90 \%$ confluency were suitable for plasmid transfection. Plasmids and Lipofectamine 2000 reagent were diluted in DMEM. The diluted plasmids were mixed with the diluted Lipofectamine 2000. The mixtures were incubated for $5 \mathrm{~min}$ at room temperature and added to cells in each dish. The transfected cells were collected after $24 \mathrm{~h}$, and the collected cells were used for further study (cell proliferation, migration and invasion). For siRNA transfection, siRNA and Lipofectamine RNAiMAX reagent were diluted in DMEM without serum, and the dilutes were mixed gently. The mixtures were incubated for 10-20 min at room temperature and then added to cells of each dish. Cells were harvested after $48 \mathrm{~h}$, and the harvested cells were used for further study (cell proliferation, migration and invasion). For the EGFR/HER2-ERK1/2 signaling pathway inhibition study, PD98059 (Sigma-Aldrich) and GW583340 (Sigma-Aldrich) were dissolved in DMSO. Cells were transfected with empty vector or pcDNA3.0-MYLK-AS1 plasmids. Twenty-four hours later, DMSO was added to cells of one group as control, and PD98059 (a final concentration of $25 \mathrm{nM}$ ) and GW583340 (a final concentration of $100 \mathrm{nM}$ ) were added to cells in other two groups, respectively. Cells were then used to perform proliferation, invasion and western blot assays.

\section{RNA isolation and reverse transcription- quantitative PCR}

Total RNA was isolated using TRIzol reagent according to the manufacturer's instructions (Invitrogen). Total RNA was reverse transcribed into first strand cDNA with oligo (dT) primers using Moloney murine leukemia virus reverse transcriptase (Promega). Then, the first strand cDNA synthesis reaction mixture was used for PCR amplification in a total volume of $50 \mu \mathrm{l}$. qPCR was performed in triplicates in a $20 \mu \mathrm{l}$ of reaction mixture containing 10 $\mu \mathrm{l}$ of SYBR Premix Ex Taq Master Mix (2×) (Takara), $0.5 \mu \mathrm{M}$ of each of the primers and $10 \mathrm{ng}$ cDNA. The relative expression was calculated by the comparative $\mathrm{Ct}$ method. Results were normalized to the expression of $\beta$-actin. Specific primer sequences were listed as follows: MYLK-AS1 forward, 5'-TTGCAGTGTTCAG
CACTGGCAC-3', reverse, 5'-ATTCGACGACCAGTG TTTCAGT-3'; $\beta$-actin forward, 5'-ATCACCATTGGC AATGAGCG-3', reverse, 5'-TTGAAGGTAGTTTCGT GGAT-3'.

\section{Cell proliferation, migration and invasion assays}

For cell proliferation assay, the transfected cells were replated into 96-well plates at 3000 cells/well. Cell proliferation was determined by the CCK-8 Kit (Dojindo Laboratories) according to the manufacturer's instructions. Briefly, ten microliters of CCK-8 solution were added to cultured cells in each well, followed by incubation at $37^{\circ} \mathrm{C}$ for $1 \mathrm{~h}$. The OD values were measured at $450 \mathrm{~nm}$ using a microplate reader. CCK-8 assay was performed after cell attachment for the first time (Day 0) and was continuously performed every $24 \mathrm{~h}$ as indicated. For colony formation assay, three thousand cells were seeded per well. Two weeks later, colonies were fixed with methanol and stained with $0.1 \%$ crystal violet (Sigma-Aldrich) and visible colonies were manually counted. Cell migration was determined using wound healing assay. Cells were seeded at a density of $4 \times 10^{4}$ cells $/ \mathrm{cm}^{2}$. On day 3 , a straight scratch was made with a $200 \mathrm{~mL}$ pipette tip and images of the wound were acquired under the microscope with the original magnification of $100 \times$. After 0 and $16 \mathrm{~h}$, cells were photographed under the microscope and the remaining scratch area was calculated. Cell invasion was determined using Transwell chambers with $8 \mu \mathrm{m}$ pore size according to the manufacturer's instructions (Millipore). To the top chamber, five thousand cells in serum-free medium were added. In the lower chamber, DMEM with 10\% FBS was added. After 16 $h$, cells that had invaded through the membrane were fixed with methanol, stained with crystal violet, and counted.

\section{Western blot analysis}

Cells and tissue samples were collected and incubated in the RIPA buffer (Sigma-Aldrich) to obtain proteins. Approximately $30 \mu \mathrm{g}$ of total proteins were loaded into each lane of $10-15 \%$ SDS-PAGE, separated in electrophoresis at a constant pressure of $160 \mathrm{~V}$ for about 1-2 $\mathrm{h}$, and transferred onto nitrocellulose membranes (Millipore). After blocking, the membranes were then incubated with primary antibodies against EGFR (Santa Cruz Biotechnology), phosph-EGFR (Santa Cruz Biotechnology), HER2 (Santa Cruz Biotechnology), RAS (Santa Cruz Biotechnology), RAF1 (Santa Cruz Biotechnology), MEK1/2 (Cell Signaling), phosph-MEK1/2 (Cell Signaling), ERK1/2 (Cell Signaling), phosph-ERK1/2 (Cell Signaling), and $\beta$-actin (Santa Cruz 
Biotechnology). $\beta$-actin was loaded as an internal reference. Bands were then treated with horseradish peroxidase (HRP)-conjugated secondary antibody (Santa Cruz Biotechnology). Bands were developed using chemiluminescence substance (Thermo Scientific). All experiments were conducted three times independently with similar results. A representative result for Western blots was provided.

\section{In vivo experiments}

The animal experiments were reviewed and approved by the Institutional Animal Care and Use Committee of the Beijing Institute of Biotechnology, Beijing, China. The nude mice (thymus deficiency/T-cell deficiency) aged 4-6 weeks were purchased from Si-Bei-Fu Biotechnology Corporation, Beijing, China. In the subcutaneous transplantation model, mice were seeded with MYLK-AS1 shRNA-expressing MHCC97-H cells or control shRNA-expressing MHCC97-H cells $\left(5 \times 10^{6}\right.$ cells suspended in $200 \mu \mathrm{L}$ of phosphate buffered saline [PBS]) in the abdominal flank. After three weeks, the mice were harvested and the tumors were collected. The tumor weights were measured by using a precision balance. The tumor length or tumor width was measured by using a Vernier Caliper. The tumor volumes were calculated as tumor length $\times$ tumor width $\times$ tumor width/2 [26,27].

\section{Statistical analysis}

Statistical significance in the preclinical experiments was assessed by two-tailed Student's $t$-test. The Kaplan-Meier method was used to evaluate overall survival of HCC patients and the log-rank test was performed to determine the differences between survival curves. Prism 7.0 (GraphPad Software) was used to analyzed all data. The statistical significance was defined as $p<0.05$ in all assays.

\section{Results}

\section{MYLK-ASI is overexpressed in HCC patients and negatively correlated with HCC prognosis}

To explore potential lncRNAs associated with the EGFR-RAS-ERK signaling pathway, we screened lncRNAs associated with EGFR in HCC using TANRIC (https://ibl.mdanderson.org/tanric/_ design/basic/main.html). Firstly, we obtained 293 lncRNAs, out of which 131 lncRNAs were positively correlated with EGFR expression. According to the value of correlation coefficient, the top 10 lncRNAs were selected for GSEA analysis. Gene sets were considered significantly enriched if the nominal p-value was less than 0.005 and the FDR less than 0.05 based on a canonical pathways gene set from the MSigDB database [28]. We identified 3 lncRNAs
(MYLK-AS1, AC135050.5 and AC118344.1) correlated with the activation of K-RAS signaling, which is a critical downstream signaling pathway of EGFR/HER2 (Figure 1A). We then compared expression levels of the 3 lncRNAs between HCC tumor tissues and adjacent normal tissues using RNA-seq data downloaded from the Cancer Genome Atlas (TCGA). Compared with corresponding normal liver tissues, HCC tumor tissues exhibited significantly upregulated expression of MYLK-AS1, but not AC135050.5 and AC118344.1 (Figure 1B). Furthermore, based on clinical information of TCGA data, we stratified HCC patients into two groups by the medians of lncRNAs expression levels. The survival analysis showed that higher expression of MYLK-AS1 was significantly correlated with poorer prognosis in HCC patients (Figure 1C). Again, the expression levels of AC135050.5 and AC118344.1 had no relations with HCC prognosis (Figure 1C). Moreover, MYLK-AS1 was not associated with K-RAS signaling activation in normal samples, which is obviously different from the GSEA result in HCC tumors (Figure S1). These data indicate that MYLK-AS1 may be involved in the activation of EGFR/HER2-ERK1/2 signaling pathway in HCC.

\section{MYLK-ASI promotes HCC cell proliferation in vitro}

To select HCC cell lines suitable for MYLK-AS1 overexpression and knockdown experiments, we used RT-qPCR analysis to examine the expression of MYLK-AS1 in four different HCC cell lines (HepG2, SMMC-7721, MHCC97-H, and BEL-7402) and one normal human hepatocyte cell line (LO2). MHCC97-H, and BEL-7402 cells expressed higher level of MYLK-AS1 than HepG2, SMMC-7721 and LO2, with LO2 expressing the lowest level of MYLK-AS1 (Figure 2A). Thus, to study the role of MYLK-AS1 in HCC, we chose MHCC97-H and BEL-7402 to knockdown MYLK-AS1 and HepG2 to overexpress MYLK-AS1. BEL-7402 and MHCC97-H cells transfected with MYLK-AS1 siRNAs grew more slowly than those transfected with control siRNA (Figure 2B), indicating that MYLK-AS1 knockdown in BEL-7402 and MHCC97-H cells reduces cell proliferation. Moreover, colony formation assays showed that knockdown of MYLK-AS1 in BEL-7402 and MHCC97-H cells reduced the colony number (Figure 2C). In contrast, HepG2 cells with overexpression of MYLK-AS1 proliferated significantly faster than those transfected with empty vector (Figure 2D). Consistently, the colony formation ability of HepG2 cells was remarkably increased by overexpression of MYLK-AS1 (Figure 2E). These results demonstrate that MYLK-AS1 promotes HCC 
cell proliferation.

\section{MYLK-AS1 accelerates migration and invasion of HCC cells in vitro}

Wound-healing assay and transwell assay were performed respectively to study the biological roles of MYLK-AS1 in the migration and invasion of HCC cells. In wound healing assay, the migration ability of BEL-7402 cells with MYLK-AS1 knockdown was significantly decreased compared to that of control cells (Figure 3A). Similar results were obtained in MHCC97-H cells. Transwell invasion assay showed that MYLK-AS1 knockdown in BEL-7402 and MHCC97-H cells decreased the number of invaded cells (Figure 3B). On the contrary, the abilities of migration and invasion of HepG2 cells were markedly promoted by overexpressing MYLK-AS1 (Figure 3C and 3D). Conclusively, our data indicate that MYLK-AS1 can facilitate migration and invasion of HCC cells.

\section{MYLK-AS1 activates EGFR/HER2-ERK1/2 signaling pathway in HCC}

The EGFR/HER2-RAS-RAF-MEK-ERK1/2 signaling pathway plays a key role in cancer development and progression. Since MYLK-AS1 correlates with the activation of K-RAS signaling, we investigated whether MYLK-AS1 modulates expression of EGFR and HER2, the K-RAS upstream regulators, as well as RAF1, MEK1/2 and ERK1/2, the K-RAS downstream targets. MYLK-AS1 knockdown in BEL-7402 and MHCC97-H cells decreased protein expression of EGFR, pEGFR, HER2 and RAF1, but not K-RAS, MEK1/2 and ERK1/2 (Figure 4A and 4B).

A

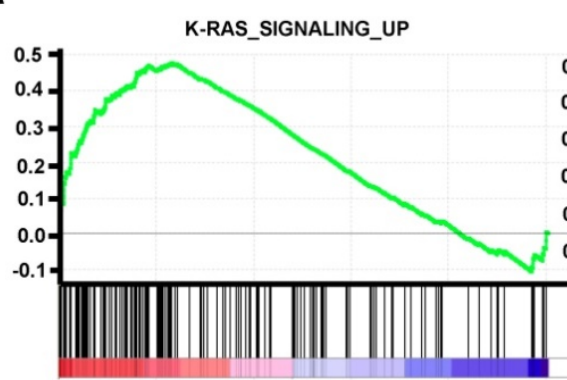

MYLK-AS1 Positively correlated Enrichment score (ES): 0.478 Nominal $P$-value: $<0.0001$ FDR $q$-value: $<0.0001$
MYLK-AS1
M

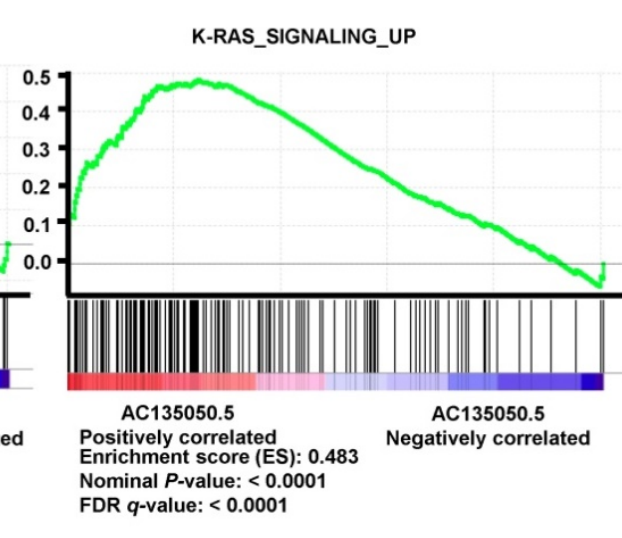

Nominal $P$-value: $<0.0001$

FDR $q$-value: $<0.0001$

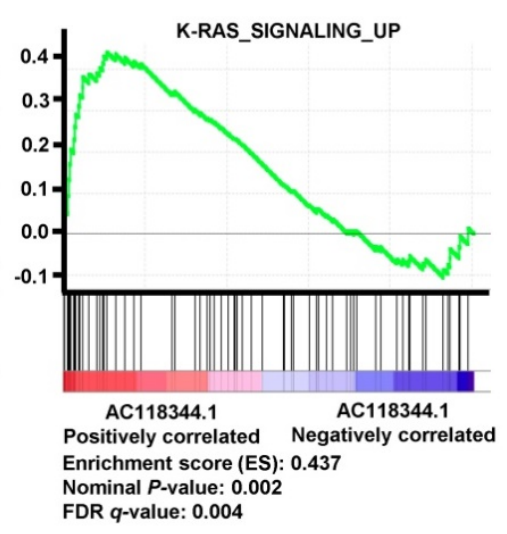

FDR $q$-value: 0.004

B
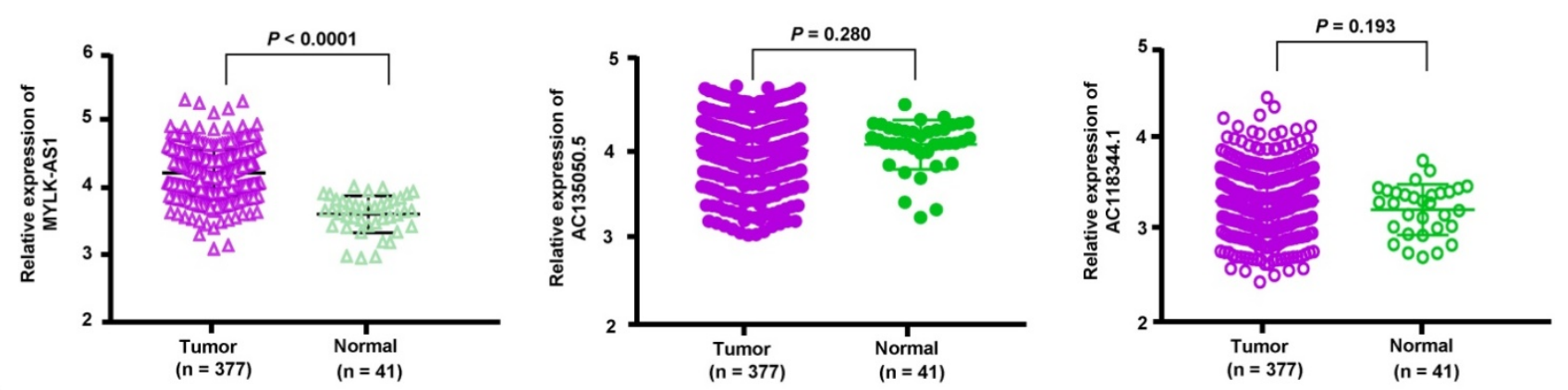

C
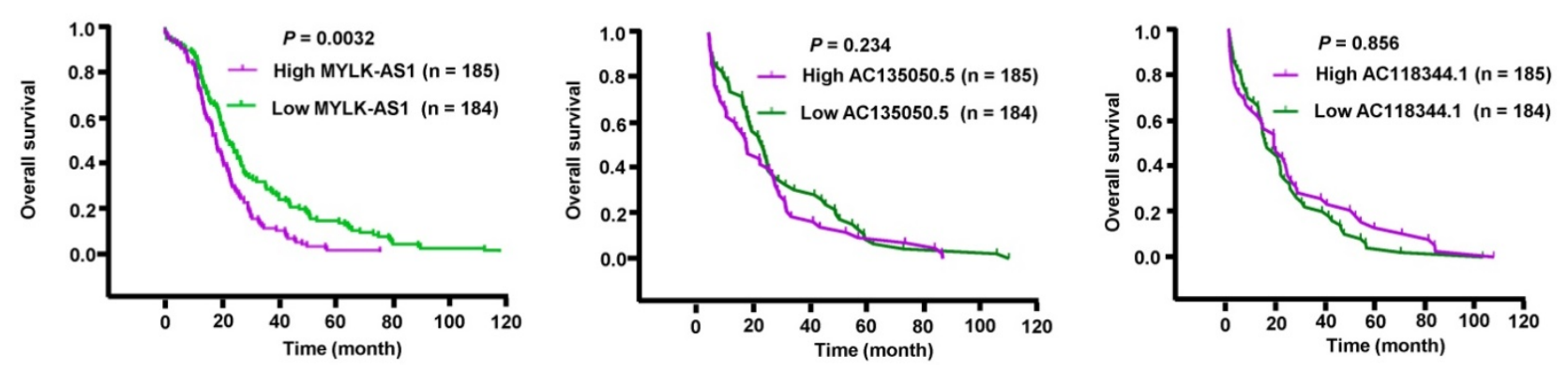

Figure 1. Analysis of HCC RNA-seq data from TCGA. (A) Cluster analysis of IncRNAs correlated with the activation of K-RAS signaling. (B) The expression levels of three IncRNAs (MYLK-AS1, AC135050.5 and AC118344.1) were compared between HCC tumor tissues and adjacent normal tissues using RNA-seq data downloaded from TCGA. (C) The relationships between the 3 IncRNAs and the HCC survival were analyzed based on clinical information of TCGA data. 
Although MYLK-AS1 knockdown did not alter MEK1/2 and ERK1/2 expression, knockdown of MYLK-AS1 reduced phosphorylation of MEK1/2 and ERK1/2, indicating that MYLK-AS1 knockdown inhibits activation of MEK1/2 and ERK1/2. Moreover, a dose dependent effect was observed when increasing amounts of MYLK siRNA were transfected into MHCC97-H cells (Figure 4B). In contrast, MYLK-AS1 overexpression in HepG2 cells increased EGFR, pEGFR, HER2 and RAF1 expression as well as phosphorylation of MEK1/2 and ERK1/2 (Figure 4C). These data suggest that MYLK-AS1 is an upstream regulatory factor of EGFR/HER2 and stimulates EGFR/HER2-ERK signaling pathway in HCC.

A

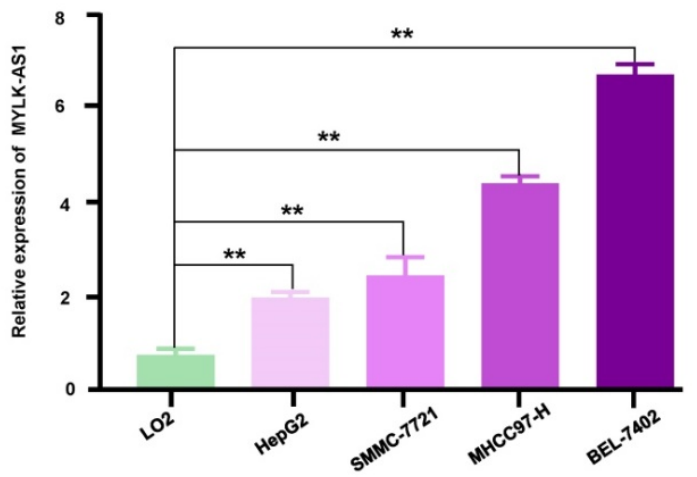

B
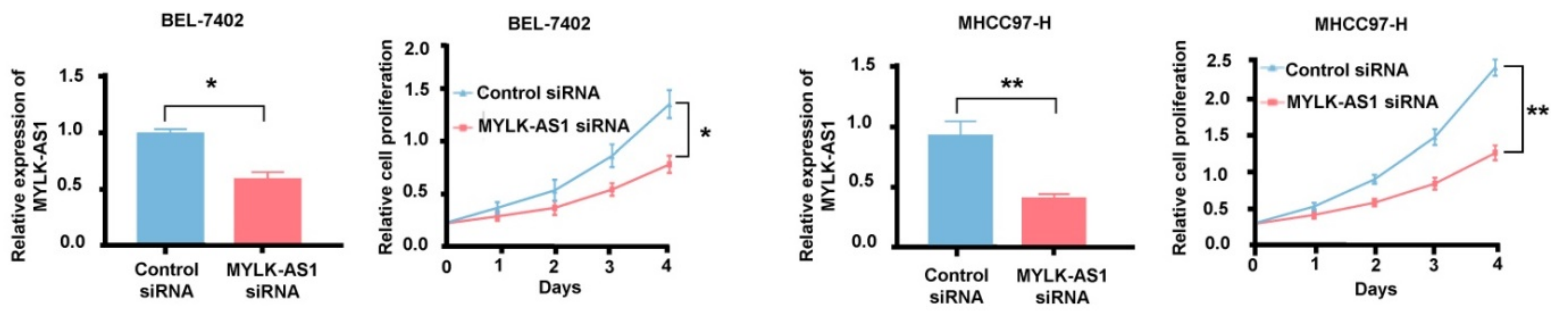

C

BEL-7402

MHCC97-H
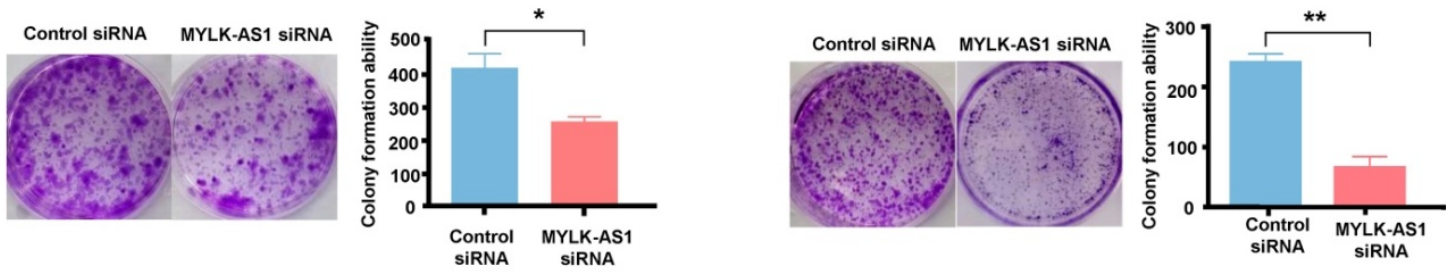

D

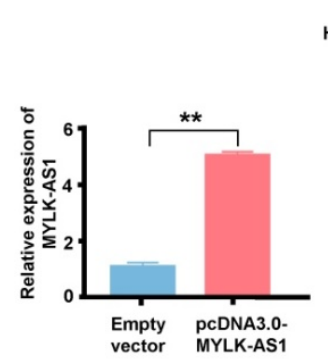

HepG2

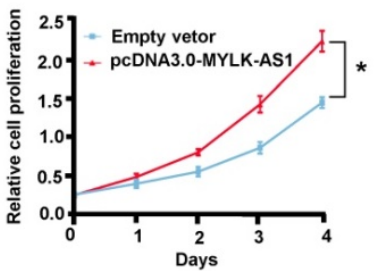

HepG2

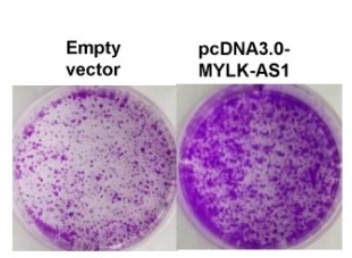

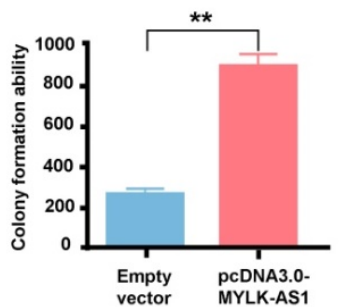

Figure 2. MYLK-AS1 increases HCC cell proliferation in vitro. (A) MYLK-AS1 expression was analyzed by RT-qPCR in normal hepatocyte cell line LO2 and HCC cell lines (HepG2, SMMC-7721, MHCC97-H and BEL-7402). (B and C) BEL-7402 and MHCC97-H cells were transfected with MYLK-AS1 siRNAs to knockdown the expression of MYLK-AS1. The knockdown effects were determined by RT-qPCR. Cell proliferation was measured by CCK-8 assay and colony formation assay. (D and E) HepG2 cells were transfected with pcDNA3.0-MYLK-AS1 to overexpress MYLK-AS1. The overexpression effects were determined by RT-qPCR. Cell proliferation was detected by CCK-8 assay and colony formation assay. All values shown are mean \pm SD of triplicate measurements and have been repeated 3 times with similar results $(* P<0.05, * * P<0.01)$. 
A
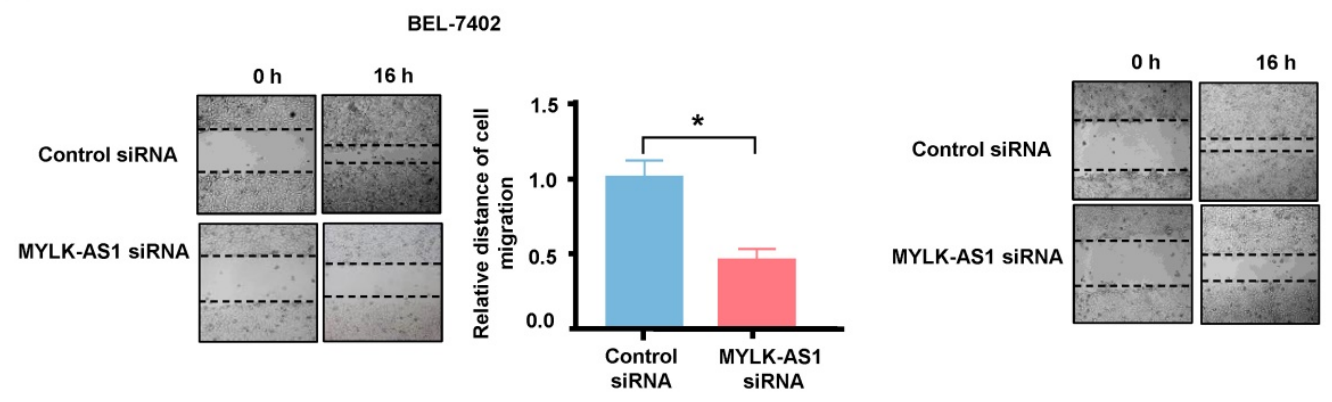

MHCC97-H

B
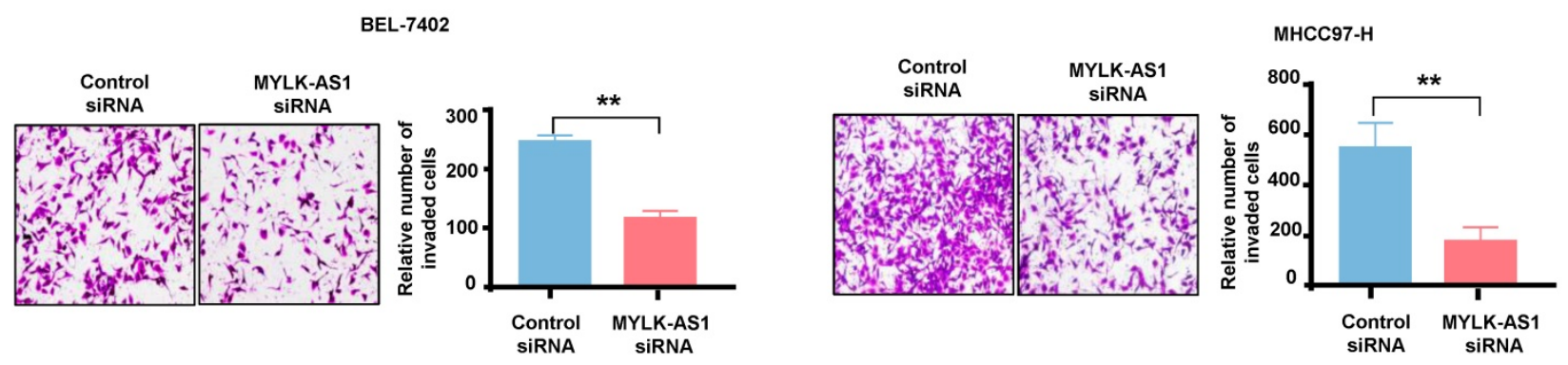

C

HepG2

D
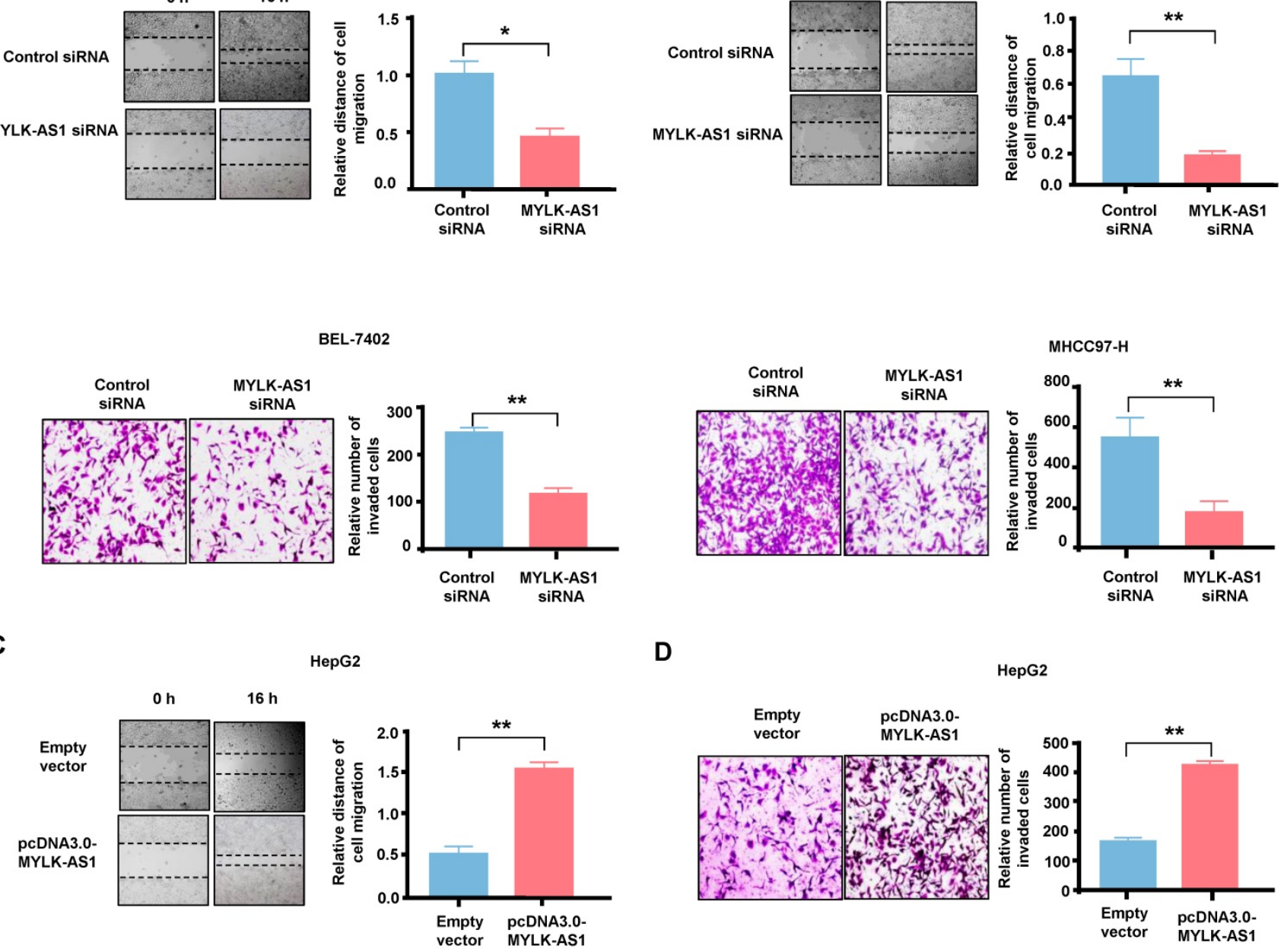

HepG2

Figure 3. The effects of MYLK-ASI on HCC cell migration and invasion in vitro. (A) BEL-7402 and MHCC97-H cells were transfected with MYLK-AS1 siRNAs to knockdown the expression of MYLK-ASI. Wound healing assay was performed to evaluate cell migration changes between control and MYLK-ASI knockdown groups in BEL-7402 and MHCC97-H cells. (B) Cells were transfected as in (A). Transwell assay was performed to investigate cell invasion differences between control and MYLK-AS1 knockdown groups in BEL-7402 and MHCC97-H cells. (C and D) HepG2 cells were transfected with pcDNA3.0-MYLK-AS1 to overexpress MYLK-AS1. Wound healing assay and transwell assay were respectively used to detect cell migration (C) and invasion (D) differences between control and MYLK-AS1 overexpression groups in HepG2 cells. Data shown are mean \pm SD of 3 independent experiments. ( $* P<0.05$ versus empty vector or control siRNA, $* * P<0.01$ versus empty vector or control siRNA).

\section{MYLK-ASI regulates proliferation and invasion of HCC cells through the EGFR/HER2-ERK1/2 signaling pathway}

To investigate the mechanism by which MYLK-AS1 regulates proliferation and invasion of HCC cells, we tested whether activation of EGFR/HER2-ERK1/2 signaling pathway is responsible for MYLK-AS1 modulation of HCC cell proliferation and invasion. As expected, the EGFR/HER2 inhibitor GW583340 and the MEK1/2 inhibitor PD98059 reduced HepG2 cell proliferation and invasion (Figure 5A and 5B). Importantly, GW583340 and PD98059 abolished the ability of MYLK-AS1 to increase HepG2 cell proliferation and invasion. Moreover, in HepG2 cells, GW583340 and PD98059 decreased phosphorylation of MEK1/2 and ERK1/2, and GW583340 reduced EGFR phosphorylation (Figure 5C), indicating that GW583340 and
PD98059 inhibit activation of MEK1/2 and ERK1/2, and GW583340 blocks activation of EGFR. Intriguingly, GW583340 and PD98059 abolished the ability of MYLK-AS1 to stimulate MEK1/2 and ERK1/2. In addition, we used ERK1/2 siRNA and EGFR siRNA to knock down the protein expressions of ERK1/2 and EGFR. Meantime, pcDNA3.0MYLK-AS1 was used to rescue the inhibitory effect of ERK1/2 and EGFR siRNAs on cell proliferation. The protein expressions of ERK1/2 and EGFR were obviously decreased by their siRNAs (Figure 5D and E). Although cell proliferation was inhibited by knocking down ERK1/2 and EGFR, overexpressing MYLK-AS1 could partially rescue the inhibitory effect (Figure $5 \mathrm{D}$ and $\mathrm{E}$ ). These results reveal that MYLK-AS1 promotes HCC cell proliferation and invasion through activating the EGFR/HER2-ERK1/2 pathway. 
A
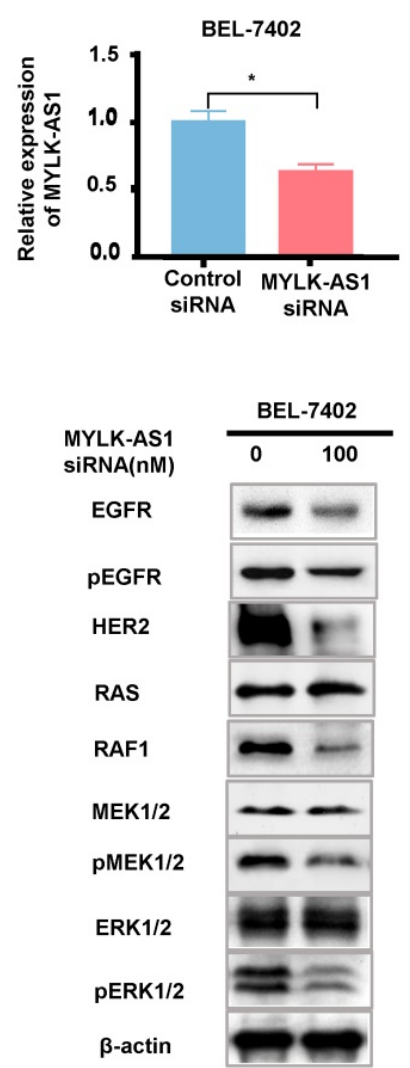

B
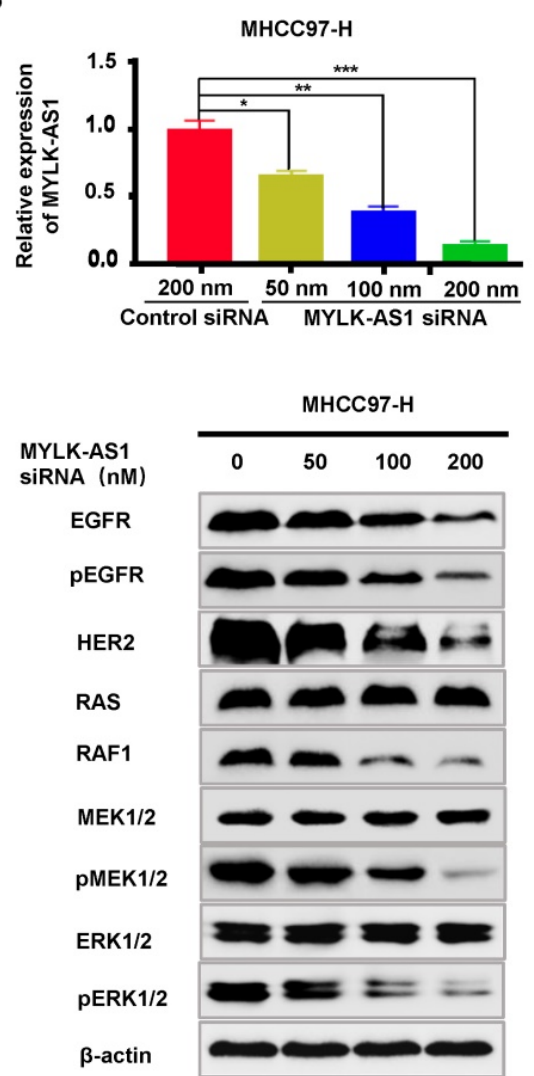

C
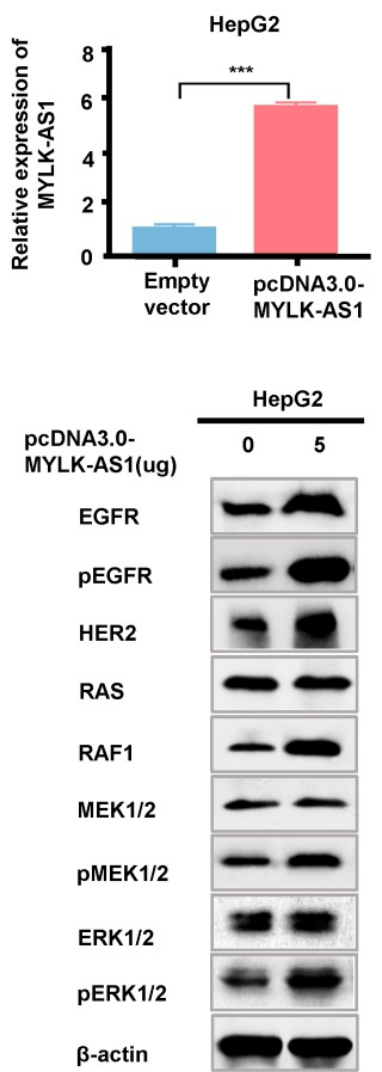

Figure 4. MYLK-AS1 activates EGFR/HER2-ERK signaling pathway in HCC. (A) BEL-7402 cells were transfected with MYLK-AS1 siRNAs (100 nM) or control siRNA $(100 \mathrm{nM})$. The MYLK-AS1 knockdown effect was detected by RT-qPCR. Western blot was performed to determine the expression of EGFR/HER2-ERK signaling pathway-related genes as indicated. $\beta$-actin was used as a loading control. (B) MYLK-AS1 siRNAs ( $50 \mathrm{nM}, 100 \mathrm{nM}$ and $200 \mathrm{nM}$ ) or control siRNA (200 nM) were transfected into MHCC $97-\mathrm{H}$ cells. The MYLK-AS1 overexpression effect was measured by RT-qPCR. Western blot was performed as in (A). (C) HepG2 cells were transfected with MYLK-AS1 (5 $\mu g$ ) or empty vector. The MYLK-ASI overexpression effect was measured by RT-qPCR. Western blot was performed as in (A). All experiments were conducted three times independently and representative immunoblot results were shown. Data were presented as the mean \pm SD $(* P<0.05, * * P<0.01)$.

\section{Knockdown of MYLK-AS1 inhibits tumor growth in vivo}

For in vivo tumor assay, the xenotransplantation model was used to further evaluate the functional effects of MYLK-AS1 on HCC. Compared to the controls, the tumor xenografts produced from MYLK-AS1 shRNA-expressing MHCC97-H cells were significantly inhibited, with smaller tumor volumes and lighter weights (Figure 6A). To further validate the molecular mechanism of MYLK-AS1 in HCC, we detected downstream molecules of EGFR/HER2-ERK signaling pathway of tumor tissues from the xenotransplantation model. As expected, the levels of EGFR, pEGFR, HER2, RAF1, and phosphorylation of MEK1/2 and ERK1/2 were markedly suppressed by downregulating MYLK-AS1 (Figure 6B). Taken together, our results illustrate that knockdown of MYLK-AS1 can inhibit HCC tumor growth in vivo.

\section{Discussion}

The EGFR/HER2 signaling pathway is frequently activated in many cancers, including hepatocellular carcinoma, lung cancer, colorectal cancer, and breast cancer, and plays a critical role in carcinogenesis and progression [29-32]. Activated EGFR or HER2 predicts poor clinical outcome of cancer patients. In line with their driver roles in cancer development and progression, cancer drugs intercepting EGFR or HER2 currently outnumber therapies targeting other hubs of signal transduction. However, drug resistance is a major obstacle in cancer treatment. Therefore, elucidating the regulation of EGFR and HER2 expression may provide new therapeutic targets for overcoming endocrine resistance or improving clinical outcomes. In this study, we show for the first time that MYLK-AS1 promotes hepatoma cell proliferation, migration and invasion in vitro, and tumor formation in nude mice, through upregulation of EGFR and HER2 as well as their downstream targets (Figure 6C). Moreover, MYLK-AS1 was upregulated in liver cancer patients and high MYLK-AS1 expression correlated with poor clinical outcome. Our data suggest that MYLK-AS1 functions as an oncogene and may be a useful molecular target for cancer therapy, especially liver cancer therapy. 
A

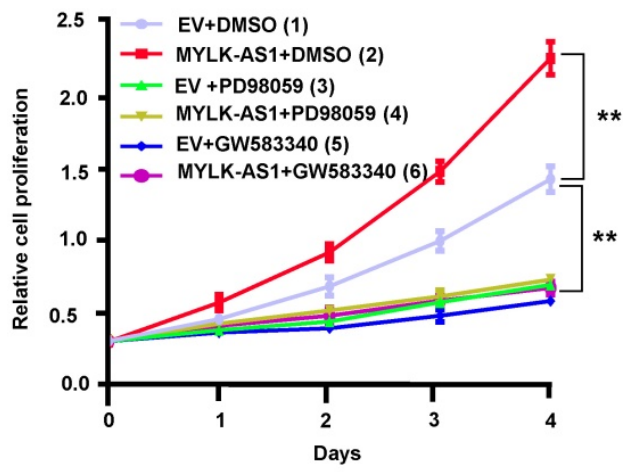

B

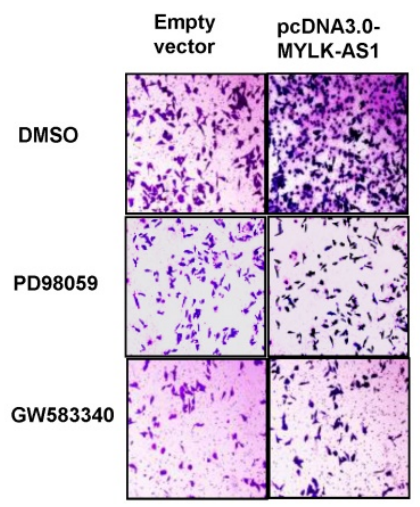

D

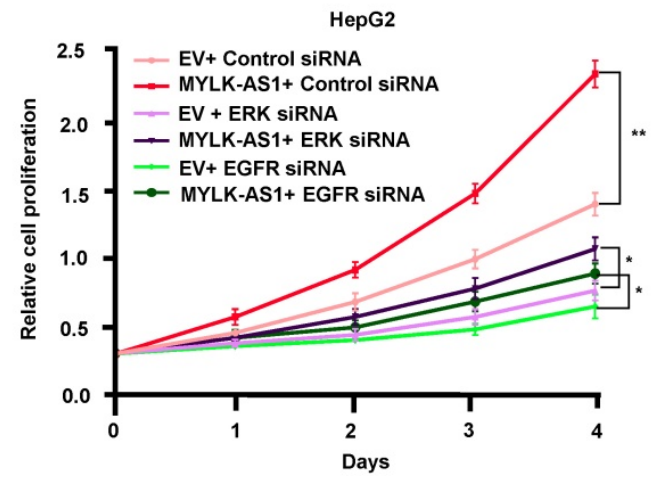

C

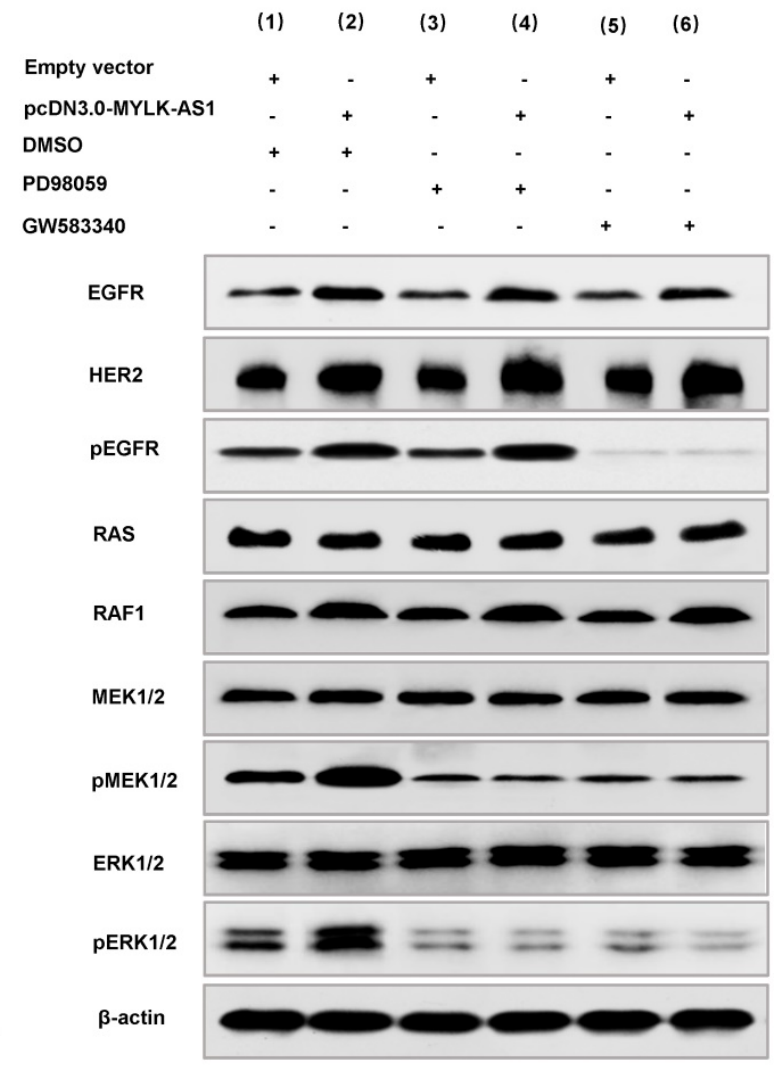

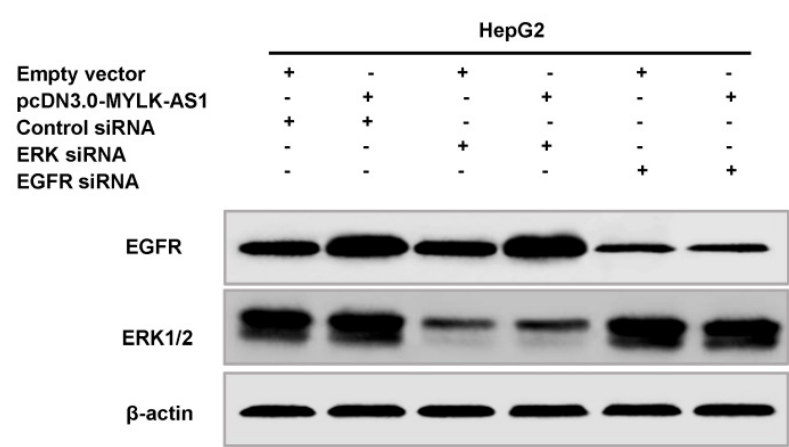

Figure 5. MYLK-AS1 regulates proliferation and invasion of HCC cells through the EGFR/HER2-ERK signaling pathway. (A) HepG2 cells were transfected with pcDNA3.0-MYLK-AS1 (MYLK-AS1) vector or empty vector (EV). Cells were treated with PD98059 or GW583340 as indicated, with DMSO as control. Cell proliferation was then determined by CCK- 8 assay. All values shown are mean \pm SD of triplicate measurements and have been repeated 3 times with similar results $(* * P<0.01$ at day 4$)$. (B) HepG2 cells were transfected as in $(A)$. The cell invasion changes were detected by transwell assay. All experiments were conducted three times independently, and the data were presented as the mean $\pm S D(* * P<0.01)$. (C) Representative immunoblot of HepG2 cells transfected as in $(A)$ with the indicated antibodies. The experiments have been repeated 3 times with similar results. (D and E) HepG2 cells were transfected with ERK siRNA or EGFR siRNA as indicated, with control siRNA as control. Twenty four hours later, the cells were transfected with pcDNA3.0-MYLK-AS1 (MYLK-AS1) vector or empty vector (EV) as indicated. After $24 \mathrm{~h}$, the transfected cells were collected and replated into new wells as indicated. Cell proliferation was then determined by CCK-8 assay for the indicated times. All values shown are mean \pm SD of triplicate measurements and have been repeated 3 times with similar results $(* * P<0.01$ at day 4$)$.

The EGFR and HER2 downstream pathways include the RAS-RAF-MEK-ERK pathway, the phosphoinosidyl-3-kinase (PI3K)/protein kinase B $(\mathrm{AKT}) /$ mammalian target of rapamycin (mTOR) pathway, the signal transducer and activation of transcription (STAT) pathway, and the phospholipase

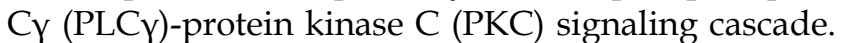

These pathways control cell proliferation, survival, differentiation, cell adhesion, migration, invasion, metastasis, angiogenesis, and metabolism. Some studies have found that lncRNAs can regulate EGFR/HER2 downstream signaling pathways via microRNAs (miRNAs). For example, lncRNA HOXA-AS3 sponges miR-29c to facilitate cell 
proliferation, metastasis, and epithelial-mesenchymal transition process, and activates the MEK/ERK signaling pathway in HCC [33]. Overexpression of lncRNA H19 attenuates miR-193b-mediated inhibition on multiple driver oncogenes (EGFR, KRAS, PTEN and IGF1R) and MAPK1 gene, and thus triggers EMT and stem cell transformation in HCC [34]. Silencing of lncRNA HOXD-AS1 inhibits proliferation, cell cycle progression, migration and invasion of HCC cells through MEK/ERK pathway [35]. We show that MYLK-AS1 not only activates
MEK/ERK, but also stimulates the expression of both EGFR and HER2, indicating that MYLK-AS1 is a key upstream regulator of EGFR and HER2. It will be interesting to investigate whether MYLK-AS1 regulates EGFR and HER2 expression through miRNAs or other molecules or whether MYLK-AS1 directly regulates the expression of EGFR and HER2. Except for the EGFR/HER2-RAS-RAF-MEK-ERK pathway, whether MYLK-AS1 modulates other above-mentioned pathways also remains to be determined.

A

Colomn $\begin{array}{llllllllll}1 & 2 & 3 & 4 & 5 & 6 & 7 & 8 & 9 & 10\end{array}$

Control shRNA MYLK-AS1 ShRNA

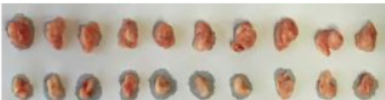

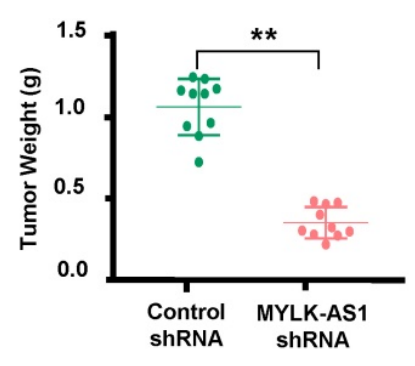

B

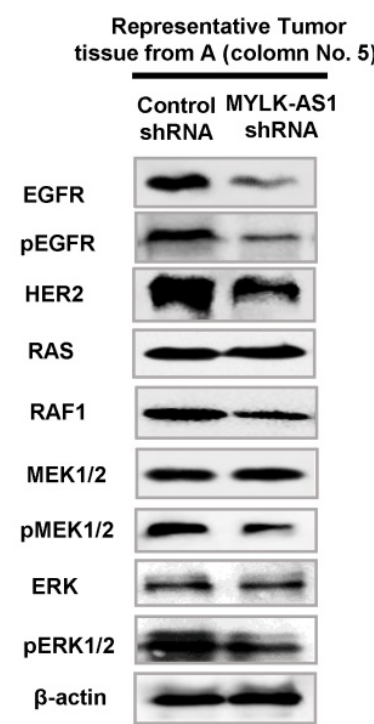

C

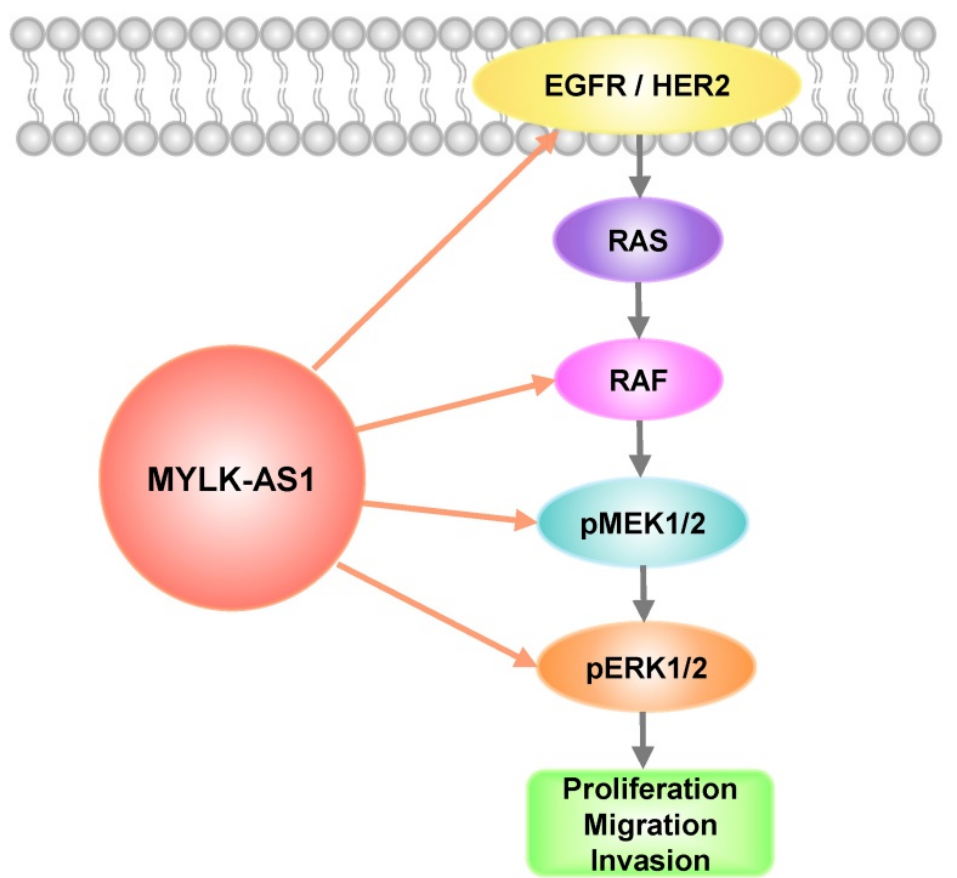

Figure 6. Knockdown of MYLK-ASI inhibits tumor growth in vivo. (A) Nude mice were seeded with MYLK-AS1 shRNA-expressing MHCC97-H cells and control shRNA-expressing MHCC97-H cells. The volume of the tumors was calculated every week after transplantation (mean \pm SD; $n=10)$. The mice were killed $21 \mathrm{~d}$ after implantation and the weight of the tumors was measured. $* * P<0.01$ at day 21 . (B) Representative immunoblot of the tumors from (A) with the indicated antibodies. (C) A proposed model underlying the role of MYLK-AS1 in hepatoma cell proliferation, migration and invasion via regulation of the EGFR/HER2-ERK1/2 pathway. 
RAF kinases are well known oncoproteins that play a key role in cancer development and progression through activation of the MEK/ERK signaling cascade [36]. MEK/ERK activation has been shown to be required for mediating the self-renewal capacity and drug-resistant properties of HCC cells, resulting in poor patient survival. Activation of the RAS/RAF/MEK/ERK signaling is present in $50 \%-100 \%$ of HCC tumors [37], yet activating mutations of RAS/RAF are infrequent. The common mechanism by which MEK and ERK are activated by RAF is the downregulation of inhibitory regulators of the pathway in HCC. Our present study showed that MYLK-AS1 dramatically decreases RAF protein expression although the underlying mechanism remains to be investigated.

In summary, our results discover the lncRNA MYLK-AS1 activating EGFR/HER2-ERK signaling pathway and disclose the pivotal role of MYLK-AS1 in promoting HCC cell proliferation, migration and invasion. In the future, exploring the exact roles of MYLK-AS1 in oncogenesis and progression as well as the underlying mechanisms will provide powerful and direct evidence to prove MYLK-AS1 as a therapeutic target for cancer.

\section{Supplementary Material}

Supplementary figure.

http://www.ijbs.com/v16p1989s1.pdf

\section{Acknowledgements}

This work was supported by grants from the National Natural Science Foundation of China (81630067, 81930078 and 81872246) and the Medical Science and Technology Youth Training Program of PLA (16QNP029).

\section{Competing Interests}

The authors have declared that no competing interest exists.

\section{References}

1. Roskoski R Jr. The ErbB/HER family of protein-tyrosine kinases and cancer. Pharmacol Res. 2014; 79: 34-74.

2. Maennling AE, Tur MK, Niebert M, et al. Molecular Targeting Therapy against EGFR Family in Breast Cancer: Progress and Future Potentials. Cancers (Basel). 2019; 11(12):1826.

3. Shao W, Zhu W, Lin J, et al. Liver X Receptor Agonism Sensitizes a Subset of Hepatocellular Carcinoma to Sorafenib by Dual-Inhibiting MET and EGFR. Neoplasia. 2019; 22:1-9.

4. Peng $S$, Wang $R$, Zhang $X$, et al. EGFR-TKI resistance promotes immune escape in lung cancer via increased PD-L1 expression. Mol Cancer. 2019; 18: 165.

5. Schlessinger J. Ligand-induced, receptor-mediated dimerization and activation of EGF receptor. Cell. 2002; 110: 669-672.

6. Yarden Y, Sliwkowski MX. Untangling the ErbB signalling network. Nat. Rev. Mol. Cell Biol. 2001; 2: 127-137.

7. Hynes NE, Lane HA. ErbB receptors and cancer: The complexity of targeted inhibitors. Nat. Rev. Cancer. 2005; 5: 341-354.

8. Nikolaos Diamantis and Udai Banerji. Antibody-drug conjugates-an emerging class of cancer treatment. Br. J. Cancer. 2016; 114: 362-367.
9. Maximiano S, Magalhães $P$, Guerreiro MP, et al. Trastuzumab in the Treatment of Breast Cancer. BioDrugs. 2016; 30: 75-86.

10. Laakmann E, Muller V, Schmidt M, et al. Systemic Treatment Options for HER2-Positive Breast Cancer Patients with Brain Metastases beyond Trastuzumab: A Literature Review. Breast Care. 2017; 12: 168-171.

11. Bose P, Ozer H. Neratinib: An oral, irreversible dual EGFR/HER2 inhibitor for breast and non-small cell lung cancer. Expert Opin Investig Drugs. 2009; 18: 1735-1751.

12. Lou $\mathrm{Y}, \mathrm{Yu} \mathrm{Y}, \mathrm{Xu} \mathrm{X}$, et al. Long non-coding RNA LUCAT1 promotes tumourigenesis by inhibiting ANXA2 phosphorylation in hepatocellular carcinoma. J Cell Mol Med. 2019; 23: 1873-1884.

13. Huang W, Liu J, Yan J, et al. LncRNA LINC00470 promotes proliferation through association with NF45/NF90 complex in hepatocellular carcinoma. Hum Cell. 2020; 33(1):131-139.

14. Bo C, Li X, He L, et al. A novel long noncoding RNA HHIP-AS1 suppresses hepatocellular carcinoma progression through stabilizing HHIP mRNA. Biochem Biophys Res Commun. 2019; 520: 333-340.

15. Song W, Zhang J, Xia Q, et al. Down-regulated lncRNA TP73-AS1 reduces radioresistance in hepatocellular carcinoma via the PTEN/Akt signaling pathway. Cell Cycle. 2019; 18: 3177-3188.

16. Siegel RL, Miller KD, Jemal A. Cancer statistics, 2018. CA Cancer J Clin. 2018; 68: 7-30.

17. Zhang Y, Li D, Jiang Q, et al. Novel ADAM-17 inhibitor ZLDI-8 enhances the in vitro and in vivo chemotherapeutic effects of Sorafenib on hepatocellular carcinoma cells. Cell Death Dis. 2018; 9: 743

18. Shao Z, Li Y, Dai W, et al. ETS-1 induces Sorafenib-resistance in hepatocellular carcinoma cells via regulating transcription factor activity of PXR. Pharmacol Res. 2018;135: 188-200.

19. Strausberg RL, Feingold EA, Grouse LH, et al. Generation and initial analysis of more than 15,000 full-length human and mouse cDNA sequences. Proc Natl Acad Sci U S A. 2002; 99:16899-903.

20. Kimura K, Wakamatsu A, Suzuki Y, et al. Diversification of transcriptional modulation: large-scale identification and characterization of putative alternative promoters of human genes. Genome Res. 2006; 16: 55-65.

21. Ye J, Zhang J, Lv Y, et al. Integrated analysis of a competing endogenous RNA network reveals key long noncoding RNAs as potential prognostic biomarkers for hepatocellular carcinoma. J Cell Biochem. 2019; 120: 13810-13825.

22. Xing $\mathrm{Y}, \mathrm{Zhao} \mathrm{Z}, \mathrm{Zhu} \mathrm{Y}$, et al. Comprehensive analysis of differential expression profiles of mRNAs and lncRNAs and identification of a 14-lncRNA prognostic signature for patients with colon adenocarcinoma. Oncol Rep. 2018; 39: 2365-2375.

23. Subramanian A, Tamayo P, Mootha VK, et al. Gene set enrichment analysis: a knowledge-based approach for interpreting genome-wide expression profiles. Proc Natl Acad Sci U S A. 2005; 102(43):15545-50.

24. Yu M, Liu X, Wu H, et al. Small interfering RNA against ERK1/2 attenuates cigarette smoke-induced pulmonary vascular remodeling. Exp Ther Med. 2017;14(5):4671-4680.

25. Yang L, Xu J, Guo L, et al. Porcine Epidemic Diarrhea Virus-Induced Epidermal Growth Factor Receptor Activation Impairs the Antiviral Activity of Type I Interferon. J Virol. 2018;92(8): e02095-17.

26. Li L, Liang Y, Kang L, et al. Transcriptional Regulation of the Warburg Effect in Cancer by SIX1. Cancer Cell. 2018; 33: 368-385.

27. Jia $\mathrm{H}$, Yang $\mathrm{Q}$, Wang $\mathrm{T}$, et al. Rhamnetin induces sensitization of hepatocellular carcinoma cells to a small molecular kinase inhibitor or chemotherapeutic agents. Biochim Biophys Acta. 2016; 1860: 1417-30.

28. J ie Li, Weida Wang, Peng Xia, et al. Identification of a five-lncRNA signature for predicting the risk of tumor recurrence in breast cancer patients. Int J Cancer. 2018; 143(9): 2150-2160.

29. Fuchs BC, Hoshida Y, Fujii $T$, et al. Epidermal growth factor receptor inhibition attenuates liver fibrosis and development of hepatocellular carcinoma. Hepatology. 2014; 59: 1577-90.

30. Wu SG, Shih JY. Management of acquired resistance to EGFR TKI-targeted therapy in advanced non-small cell lung cancer. Mol Cancer. 2018; 17(1):38

31. Okada Y, Kimura T, Nakagawa T, et al. EGFR Downregulation after Anti-EGFR Therapy Predicts the Antitumor Effect in Colorectal Cancer. Mol Cancer Res. 2017;15(10):1445-1454.

32. Kjaer IM, Bechmann T, Brandslund I, et al. Prognostic and predictive value of EGFR and EGFR-ligands in blood of breast cancer patients: a systematic review. Clin Chem Lab Med. 2018; 56(5):688-701.

33. Tong Y, Wang M, Dai $Y$, et al. LncRNA HOXA-AS3 Sponges miR-29c to Facilitate Cell Proliferation, Metastasis, and EMT Process and Activate the MEK/ERK Signaling Pathway in Hepatocellular Carcinoma. Hum Gene Ther Clin Dev. 2019; 30: 129-141.

34. Ye Y, Guo J, Xiao P, et al. Macrophages-induced long noncoding RNA H19 up-regulation triggers and activates the miR-193b/MAPK1 axis and promotes cell aggressiveness in hepatocellular carcinoma. Cancer Lett. 2020; 469:310-322.

35. Sun J, Guo Y, Bie B, et al. Silencing of long noncoding RNA HOXD-AS1 inhibits proliferation, cell cycle progression, migration and invasion of hepatocellular carcinoma cells through MEK/ERK pathway. J Cell Biochem. 2020; 121: 443-457.

36. Lavoie $\mathrm{H}$, Therrien M. Regulation of RAF protein kinases in ERK signalling. Nat Rev Mol Cell Biol. 2015; 16:281-98. 
37. Ito $\mathrm{Y}$, Sasaki $\mathrm{Y}$, Horimoto M, et al. Activation of mitogen-activated protein kinases/extracellular signal-regulated kinases in human hepatocellular carcinoma. Hepatology. 1998; 27: 951-8. 